\title{
Insights Into the Biological Role of NEDD4L E3 Ubiquitin Ligase in Human Cancers
}

\author{
Shangdan Xie, Lu Xia, Yizuo Song, Hejing Liu, Zhi-wei Wang ${ }^{*}$ and Xueqiong Zhu ${ }^{*}$ \\ Department of Obstetrics and Gynecology, The Second Affiliated Hospital of Wenzhou Medical University, Wenzhou, China
}

Neural precursor cell expressed developmentally downregulated 4-like (NEDD4L) is an E3 ubiquitin ligase that has been reported to participate in multiple cellular procedures by regulating of substrate ubiquitination and subsequent protein degradation. A great amount of evidence has demonstrated that NEDD4L mainly functions as a tumor

OPEN ACCESS

Edited by:

Carlos Pérez-Plasencia, National Autonomous University of Mexico, Mexico

Reviewed by:

Omar Khan,

Hamad bin Khalifa University, Qatar

Vijay Menon,

Yale University, United States

*Correspondence: Zhi-wei Wang zhiweichina@126.com Xueqiong Zhu

zjwzzxq@163.com

Specialty section:

This article was submitted to Molecular and Cellular Oncology,

a section of the journal

Frontiers in Oncology

Received: 12 September 2021

Accepted: 28 October 2021

Published: 15 November 2021

Citation:

Xie S, Xia L, Song Y, Liu H, Wang Z-W and Zhu X (2021) Insights Into the

Biological Role of NEDD4L E3 Ubiquitin Ligase in Human Cancers.

Front. Oncol. 11:774648.

doi: 10.3389/fonc.2021.774648 suppressor in most cancer types, while it also acts as an oncogene in a few cancers. In this review, we summarize the potential role of NEDD4L in carcinogenesis and the related underlying molecular mechanism to improve our understanding of its functions in the tumorigenesis of human malignancies. Developing clinical drugs targeting NEDD4L could be a potential therapeutic strategy for cancer therapy in the future.

Keywords: NEDD4L, ubiquitination, cancer, treatment, degradation

\section{INTRODUCTION}

Ubiquitination is a cellular biological process that specifically modifies posttranslational proteins and results in substrate degradation, stabilization or relocation (1). Several successive enzyme reactions constitute a cascade of ubiquitination. Ubiquitin was originally linked to E1 ubiquitin activating enzymes for its activation in an ATP-dependent manner and then instantaneously shifted to E2 ubiquitin conjugating enzymes. Afterward, the transfer of ubiquitin from E2 to the substrate is mediated by E3 ubiquitin protein ligases (E3s) $(2,3)$. Abnormalities of ubiquitination have been confirmed to be related to tumor occurrence and development (4). It is known that E3s determine the substrate specificity of the system to a large extent (5); therefore, the disorders of E3s might lead to the initiation of human cancer.

To date, there have been more than $600 \mathrm{E} 3 \mathrm{~s}$ in the human genome, which are mainly sorted into three categories: really interesting new gene (RING) finger family E3s, the RING-between-RING (RBR) family E3s, and homologous to the E6-AP C-terminus (HECT) family E3s (6). HECT family E3s have an active site for cysteine, where cysteine binds to ubiquitin for an intervening thioester prior to its substrate (7). Specific recognition of substrates is based on the nonconservative N-terminus of HECT family E3s (8). As the most familiar and studied group of HECT family E3s, the neural precursor cell expressed developmentally downregulated 4 (NEDD4) subfamily has nine members, including NEDD4 (known as NEDD4-1), NEDD4-like (NEDD4L, also named NEDD4-2), ITCH, Smurf1, Smurf2, WWP1, WWP2, NEDL1 (also named HECW1) and NEDL2 
(also named HECW2), and is characterized by a WW domain and a C2 domain $(9,10)$. NEDD4-like, is a member of the NEDD4 subfamily and is reported to regulate various ion channels and virus budding (11). NEDD4L plays a potential role in the growth of the central nervous system, the regulation of hypertension and the development of cancer and so on (12). In recent years, compelling evidence has shown that NEDD4L accelerates or weakens the progression of various types of cancers by targeting different substrates $(13,14)$. As a result, this review presents the structure of NEDD4L and aims to summarize the function of NEDD4L in diverse cancer types.

\section{STRUCTURE AND FUNCTION OF NEDD4L}

In the NEDD4 ubiquitin ligase family, NEDD4L is the most analogous homolog of NEDD4, the archetypal member of the family (15). NEDD4L is widely distributed and highly conserved in vertebrates (11). Human NEDD4L is located on chromosome $18 \mathrm{q} 21.31$ and has 41 exons. NEDD4L exists as two protein bands in various tissues, including human tissue, one of which changes marginally due to tissue specificity, and the other one, a stably expressed protein, containing an N-terminal C2 domain, $4 \mathrm{WW}$ domains and a C-terminal HECT domain (Figure 1) (11). The chief function of the $\mathrm{C} 2$ domain consists of $\mathrm{Ca}^{2+}$ binding, membrane targeting and protein-protein interactions (16). The WW domains play a pivotal role in discriminating and provoking the specific substrates of NEDD4L (17). The HECT domain contains the catalytic cysteine 42 and participates in catalyzing polyubiquitin chain packaging, a two-step mechanism implicated in two E2 ubiquitin binding sites (18). The C2 domain and the HECT domain restrain each other to control their own activity in normal circumstances (19). This state of equilibrium is broken when intracellular $\mathrm{Ca}^{2+}$ binds to the $\mathrm{C} 2$ domain, thus stimulating the ubiquitin ligase activity of NEDD4L and then recruiting it to the plasma membrane (20). Expectedly assembling substrate-linked ubiquitin chains, including Lys-63, Lys-48, Lys-27, Lys-11 and Lys-6 linkages, NEDD4L can result in substrate degradation by lysosomes or the proteasome, and/or change the cell signaling pathway $(18,21,22)$.
The original and most familiar function of NEDD4L is to regulate the epithelial $\mathrm{Na}^{+}$channel $(\mathrm{ENaC})$, which is closely related to the fluctuation of blood pressure (23). In addition, NEDD4L also participates in DNA repair, antiviral immunity and tumor development (24-26). To date, multiple reports have shown that the expression of NEDD4L in cancers is abnormal, and various proteins have been validated to bind with NEDD4L or be ubiquitinated by NEDD4L, thus modulating the cancer development (27). For instance, DNA damage-binding protein 2 (DDB2) suppresses the expression of NEDD4L and then affects the transforming growth factor- $\beta$ (TGF- $\beta$ ) signaling in ovarian cancer (28). This review focuses on the potential role and related molecular mechanisms of NEDD4L in carcinogenesis and tumor progression.

\section{THE INVOLVEMENT OF NEDD4L IN DIFFERENT SIGNALING PATHWAYS}

NEDD4L is implicated in the regulation of various signaling pathways. For example, NEDD4L induced the ubiquitination of Unc51-like kinase 1 (ULK1), an autophagy initiation related protein, to control autophagy $(29,30)$. Endoplasmic reticulum stress increases the level of NEDD4L to trigger autophagy (31). 8Oxoguanine DNA glycosylase (OGG1) is the central cellular enzyme applied in the excision of 8-oxoguanine DNA base lesions in DNA via the base excision repair pathway, and NEDD4L is involved in OGG1 ubiquitylation in response to DNA damage (24). In addition, NEDD4L catalyzed Dishevelled 2 (Dvl2) polyubiquitination, and Dvl2 was considered as a major mediator of both $\mathrm{Wnt} / \beta$-catenin and Wnt/planar cell polarity pathways (21), suggesting that NEDD4L inhibited Wnt signaling. In addition, NEDD4L mediated the ubiquitination of PIK3CA and then weakened PI3K-AKT signaling (32). It was also reported that NEDD4L might enhance MAPK/ERK signaling but few studies have focused on concrete mechanisms (13). The specific recognition of the TGF- $\beta$ triggered phosphoThr-ProTyr motif in the junction region by the WW domain of NEDD4L contributed to Smad2/3 polyubiquitination and degradation, thus inhibiting TGF- $\beta$ signaling (33).

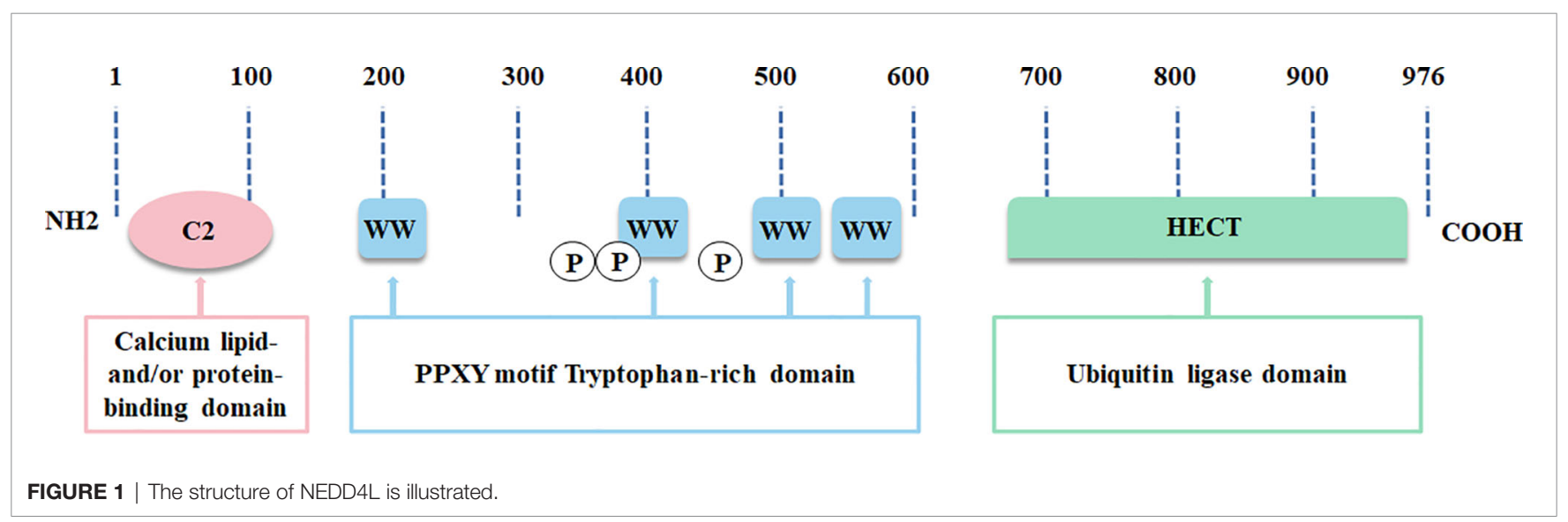




\section{THE FUNCTION OF NEDD4L IN CANCER}

\section{Gastric Cancer}

Gastric cancer ranks the fifth among the most common cancers and is the third-leading cause of cancer death (34). To date, it has been reported that the expression of NEDD4L is positively related to the outcomes of gastric cancer patients (35). Patients with negative NEDD4L expression tended to have lymphatic infiltration, metastasis and vascular invasion in sharp contrast to those with positive NEDD4L expression (35). In addition, a high level of NEDD4L generally corresponded to a low level of HIF$1 \alpha$ in gastric cancer tissues and led to a favorable prognosis (36). Collectively, NEDD4L might affect the metastasis of gastric cancer and together with HIF- $1 \alpha$, could predict the prognosis of patients. However, further experiments at the cellular and animal levels are needed to test the function of NEDD4L in gastric cancer development.

\section{Liver Cancer}

Liver cancer, as the second most lethal cancer worldwide, emerges in approximately 900, 000 people and causes about 830,000 patient deaths every year (37). In hepatocellular carcinoma (HCC), the $\mathrm{Wnt} / \beta$-catenin signaling pathway is frequently abnormally regulated (38). A study showed that the expression of NEDD4L was elevated when Wnt/ $\beta$-catenin was activated in HCC (39). However, another study revealed that the decreased expression of NEDD4L could enhance the proliferation ability of HCC cells (13). In an in vivo experiment, overexpression of NEDD4L attenuated the growth of xenograft tumors in nude mice (13). In addition, both of in vivo and in vitro experiments suggested that NEDD4L might advance the MAPK/ERK signaling pathway to weaken the proliferation of HCC cells via the induction of apoptosis (13). In patients, it was found that the expression of NEDD4L in HCC tissues was lower than that in paracancerous tissues and the patients with high NEDD4L expression had better outcomes than those with low expression of NEDD4L (13). Consequently, NEDD4L might act as a tumor-suppressor gene to repress the malignant biological behavior of HCC, while the relationship between NEDD4L and the Wnt/ $\beta$-catenin signaling pathway needs further investigation to prove.

\section{Colorectal Cancer}

Colorectal cancer (CRC) is the third most frequent cancer and the second most deadly cancer globally (37). By inhibiting the destruction of the LGR5 receptor, the absence of NEDD4L could facilitate the signal transduction of $\mathrm{Wnt} / \beta$-catenin and the number of intestinal stem cells, thus promoting the susceptibility and progression of colorectal tumors (40). Moreover, the expression of NEDD4L was all reduced in various stages of colorectal cancer specimens, in contrast to that in adjacent normal mucosal tissues. NEDD4L significantly suppressed the $\mathrm{Wnt} / \beta$-catenin signaling pathway in CRC cells (41). NEDD4L can degrade serine/threonine kinase 35 (STK35) by ubiquitination and then inhibit glycolysis, increasing the apoptosis of CRC cells by suppressing the Akt signaling pathway and modulating the chemoresistance of CRC cells (42).
In addition, a study revealed that the expression of NEDD4L in rectal cancer patients was upregulated after radiotherapy, suggesting that NEDD4L might be helpful for the treatment of rectal cancer patients (43). Taken together, NEDD4L possibly weakens the development of CRC and more in vivo experiments are required to validate the function of NEDD4L.

\section{Pancreatic Cancer}

Pancreatic cancer is a malignant cancer with a poor prognosis and takes approximately 466,000 lives away every year (37). Mounting studies have discovered that NEDD4L plays a pivotal role in the development of pancreatic cancer. MicroRNA-23A (miR-23A) inhibits the expression of NEDD4L to collaborate with miR-21 and miR-27A, thus promoting the progression of pancreatic cancer $(44,45)$. NEDD4L was demonstrated to be linked to iron metabolism in pancreatic cancer. $\mathrm{N}$-myc downstream regulated gene-1 (NDRG1), an iron-regulated metastasis suppressor, upregulated the expression of NEDD4L in PANC1 pancreatic cancer cells (46). NEDD4L mediates the degradation of the ironbinding transport protein lactotransferrin (LTF) by ubiquitination to hinder the malignant biological behavior of pancreatic cancer (47). Besides, NEDD4L impeded autophagy and cancer cell proliferation by being involved in the degradation of ULK1, and weakening the expression of the glutamine transporter ASCT2 by the ubiquitination pathway in pancreatic cancer (30). Hence, NEDD4L acts as a tumor-suppressor gene in pancreatic cancer.

\section{Gallbladder Cancer}

Although gallbladder cancer is an uncommon cancer, delayed diagnosis and poor prognosis are distinctive features (48). A study demonstrated that the expression of NEDD4L in the cytoplasm of invasive cancer cells is much higher than that in normal or dysplastic epithelial cells (14). Furthermore, NEDD4L enhanced the invasion ability of gallbladder carcinoma cells by increasing metalloproteinase-1 (MMP-1) and MMP-13 expression (14). Interestingly, downregulation of NEDD4L did not affect cell growth in gallbladder cancer (14). Unlike with most cancer types, NEDD4L might exert a tumor-promoting effect on gallbladder cancer.

\section{Lung Cancer}

Lung cancer is the main cause of cancer death and seriously threatens the lives of people worldwide (49). It was reported that NEDD4L is considered as one of the central drivers in lung adenocarcinoma (LUAD) and might affect the prognosis of the patients (50). Downregulation of NEDD4L was found in nonsmall cell lung cancer (NSCLC) samples in contrast to those of normal tissues. In NSCLC patients, NEDD4L rs11660748 $\mathrm{A}>\mathrm{G}$ and $\mathrm{rs73440898} \mathrm{A}>\mathrm{G}$ had adjusted hazard ratios (HRs) of 1.31 and 1.27, respectively, for overall survival, which means that the mutations at these two sites might impair the prognosis of patients. Moreover, low expression of NEDD4L was prone to lymph node invasion, late stage and deprived prognosis $(51,52)$. These results suggest that NEDD4L might inhibit the progression of lung cancer. Further studies demonstrated that NEDD4L suppressed the proliferation, 
migration and invasion of lung cancer cells (51). Enhancer of zeste homolog 2 (EZH2) is a highly conserved histone methyltransferase (HMTase) and is abnormally expressed in various cancers (53). Notably, EZH2 could weaken the expression of NEDD4L to serve as an oncogene in lung cancer (51). In addition, miR-93 is a member of the miR-106b-25 cluster and a driving force for the development of numerous cancers, such as bladder cancer, prostate cancer and lung cancer (54-56). MiR-93 mediated the decrease in NEDD4L expression and then enhanced TGF- $\beta$ signaling to activate epithelial-to-mesenchymal transition (EMT) in lung cancer (57). Tumor-associated macrophages (TAMs) play a pivotal role in tumor aggressiveness and M2 macrophage-derived exosomes (MDEs) are critical communication media in the tumor microenvironment (50). MDE was reported to reduce the expression of NEDD4L and then stabilize the c-Myc protein to induce chemoresistance in lung cancer by transferring miR3679-5p to cancer cells (58). In addition, NEDD4L be involved in the ubiquitination of multidrug resistance-associated protein 1 (MRP1), which was negatively correlated with the prognosis of patients with lung cancer (59). However, NEDD4L might act as an oncogene to some extent. NEDD4L could repress the expression of general control nonderepressible kinase 2 (GCN2) to control its proapoptotic effect on lung cancer cells (60). Overexpression of GCN2 aggravated cell apoptosis that was induced by $\mathrm{Na}^{+}, \mathrm{K}^{+}$-ATPase ligand in A549 lung cancer cells, indicating that NEDD4 might repress apoptosis of lung cancer cells by targeting GCN2 (60). In summary, NEDD4L could inhibit the progression of lung cancer by targeting multiple signaling pathways, but might suppress the apoptosis of cancer cells to a certain extent.

\section{Nasopharyngeal Carcinoma}

Nasopharyngeal carcinoma is an epithelial carcinoma that is closely related to the Epstein -Barr virus (EBV) infection. There are approximately 133,000 new cases and 80,000 deaths worldwide every year (37). A study utilized whole-exome capture/sequencing in 251 patients with different EBV infections (205 affected, 21 obligate carriers and 25 unaffected) and revealed that NEDD4L might regulate EBV infection (61). However, the mechanism of modulating EBV infection by NEDD4L and the role of NEDD4L in the initiation and progression of nasopharyngeal carcinoma are poorly understood. Further studies are needed to verify the function of NEDD4L in nasopharyngeal carcinoma.

\section{Ovarian Cancer}

Ovarian cancer is one of the top 10 most common cancers in females, with an approximately $46 \%$ five-year survival rate (62). The expression of NEDD4L was reduced in invasive ovarian cancer tissues in sharp contrast to that in normal ovarian epithelial tissues (63). Furthermore, the patients with higher levels of NEDD4L tended to have an early clinical stage, few lymph node metastases and good survival (63). However, NEDD4L might exert a tumor-promoting effect, and the expression of NEDD4L was downregulated by DNA damagebinding protein 2 (DDB2) (28). DDB2 participates in many biological processes including gene transcription and cell cycle regulation and is identified as a critical factor in tumor development $(64,65)$. DDB2 could attenuate the expression of NEDD4L and then stimulate TGF- $\beta$ signaling to inhibit the proliferation of ovarian cancer cells (28). These data suggested that TGF- $\beta$ might be a potential substrate of NEDD4L. However, the mechanism of inhibition of NEDD4L on TGF- $\beta$ signaling was not investigated in this study; thus, more studies are needed to verify the relationship between NEDD4L and TGF- $\beta$. Few studies have investigated the independent effect of NEDD4L on ovarian cancer, and more research needs to be carried out.

\section{Endometrial Cancer}

Endometrial cancer is the sixth most common cancer in women, and the incidence and mortality of this cancer have increased in recent years (59). Endometrial cancer tissues showed decreased expression of NED44L compared with endometrial hyperplasia tissues utilizing immunohistochemical staining (66). However, there is a lack of research investigating the relationship between the level of NEDD4L and the initiation, development and outcomes of endometrial cancer.

\section{Prostate Cancer}

The expression and functions of NEDD4L in prostate cancer are still ambiguous. The expression of NEDD4L was decreased in prostate cancer specimens compared with benign prostatic hyperplasia (67). Interestingly, the expression of three NEDD4L transcripts, NEDD4Lf, NEDD4Lg and NEDD4Lh, was upregulated in prostate cancer cells after androgen administration $(68,69)$. NEDD4L was also downregulated in androgen-independent prostate cancer cells (70). It was suggested that the dysregulation of NEDD4L might result from the level of androgen. Additionally, SOX5 and DNA methylation possibly acted as regulators of NEDD4L in androgenindependent cancer cells (70). However, the study failed to show the molecular mechanisms by which NEDD4L modulated prostate carcinogenesis. Unexpectedly, Hellwinkel et al. reported that the level of NEDD4L was higher in prostate cancer tissues than in adjacent normal tissues (71). Furthermore, NEDD4L might contribute to the development of prostate cancer by reducing the TGF- $\beta$ signaling pathway (71). Hence, the function of NEDD4L in prostate cancer is not clear.

\section{Renal Cancer}

Renal cell carcinoma (RCC) is a common malignant tumor in the urinary system (37). It was reported that the expression of NEDD4L was positively related to overall survival and diseasespecific survival (DSS) in clear cell renal cell carcinoma (ccRCC) and chromophobe cell renal carcinoma (CCRC) $(72,73)$. In further studies, NEDD4L could limit the proliferation and metastasis of ccRCC cells by weakening the ERBB3 and MAPK signaling pathways (73). Consequently, NEDD4L can be considered as a potential therapeutic target in ccRCC.

\section{Breast Cancer}

Breast cancer is the most common malignancy in females, and hormones, reproduction and lifestyle may affect the 
tumorigenesis of breast cancer (74). It was reported that a high level of NEDD4L might indicate a beneficial prognosis with free recurrence (75). In various breast cancer cells, miR-106b-25 could inhibit the expression of NEDD4L to upregulate the level of NOTCH1, which was instrumental in tumor-initiating cell (TIC) induction $(75,76)$. In addition, pseudokinase Tribble 3 (TRIB3) interacted with Akt and repressed NEDD4L-mediated ubiquitination of Forkhead box O1 (FOXO1) and then promoted the expression of Sry-related high-mobility box 2 (SOX2), a transcription factor of cancer stem cells, to exacerbate the development of breast cancer stem cells (77). NEDD4L modulated the degradation of copper transporter 1 (CTR1) in ubiquitination and exerted tumor inhibition through the CTR1Akt signaling pathway (78). Several studies found that NEDD4L was probably implicated in the anticancer or cancer-promoting effects of some substances in breast cancer. For instance, selenium (Se) is a lurking anticancer nutrient (79), and NEDD4L is considered as a key gene in the regulatory network of the Se-stimulated epigenome (80). Unexpectedly, Orai3 advanced calcium influx, activated NEDD4L and reduced the apoptosis of breast cancer cells by weakening the expression of p53 (81). The relationship between NEDD4L and p53 has been reported, and NEDD4L might promote the survival of cancer cells (82). In brief, most research suggests that NEDD4L suppresses the malignant biological behavior of breast cancer cells, and further studies are necessary to discover its underlying mechanism in breast carcinogenesis.

\section{Other Human Cancers}

NEDD4L, activated by a functional polypeptide (JP1), induced degradation of SP1 via the ubiquitin-proteasome pathway and then attenuated the transcription of integrin $\alpha v \beta 3$ to inhibit cell proliferation and metastasis of melanoma (83). In addition, serum- and glucocorticoid-regulated kinase 1 (SGK1) inhibited the degradation of the transcription factor JunB by NEDD4L to enhance $\mathrm{T}_{\mathrm{H}} 2$ differentiation (84). The number of lung tumors in SGK1-deficient mice was much less than that in control mice after injection of melanoma cells into the tail vein (84). This finding indicated that NEDD4L might exert antitumor effects in melanoma. However, a study found that the expression of NEDD4L in cutaneous melanoma and lymph node metastatic melanoma was higher than that in normal melanocytes or benign nevus tissue (85). Overexpression of NEDD4L promoted the growth of A2058 melanoma cells in vivo, and downregulation of NEDD4L reduced the growth of G361 melanoma cells in vitro (85). Therefore, the role of NEDD4L in the development of melanoma is controversial and needs to be further clarified.

The expression of NEDD4L was in negatively correlated with the pathological grade of malignant glioma, and low expression of NEDD4L indicated poor outcomes (86). In addition, NEDD4L was reported as a direct target of miR-513a-5p, delayed the growth of glioma cells and amplified the cytotoxicity of temozolomide (TMZ) (87). Moreover, NEDD4L mediates the ubiquitination of the tumor oncogene sphingosine kinase 2 (SphK2) and thus suppresses the development of malignant glioma (88). Consequently, NEDD4L mainly crippled the malignant biological behavior of glioma. The role of NEDD4L in lymphoma has also been investigated. A study demonstrated that the expression of NEDD4L in Sezary syndrome (SS) was much higher than that in healthy controls (89). It was reported that NEDD4L might be involved in the progression of diffuse large B cell lymphoma (DLBCL) (90). Nevertheless, the specific effect of NEDD4L on lymphoma has not been confirmed. NEDD4L was found to be downregulated and correlated with biosynthesis and metabolism in clear-cell renal cell cancer (ccRCC) by integrated bioinformatics analysis. Low expression of NEDD4L was associated with dismal prognosis in ccRCC, suggesting that NEDD4L could act as a prognostic biomarker and therapeutic target in ccRCC (72). A bioinformatics analysis revealed that NEDD4L was downregulated in esophageal cancer patients and might be associated with esophageal cancer prognosis (91).

\section{TARGETING NEDD4L FOR CANCER THERAPY}

Emerging evidence has demonstrated that several compounds can target the expression of NEDD4L. For example, $\beta, \beta$-dimethyl-acrylalkannin (ALCAP2), a natural small-molecule compound separated from the root of Lithospermum erythrorhizon, could increase the expression of NEDD4L to hinder the nuclear translocation of $\beta$ catenin and stimulate the conjunction of ubiquitin and $\beta$-catenin, thus blocking the Wnt signaling pathway and inhibiting cell proliferation and metastasis as well as inducing apoptosis and cell cycle arrest in LUAD (92). Dexamethasone, a pretreatment drug, has been used for cancer treatment to reduce the toxic effect of chemotherapy. Dexamethasone was reported to promote the lung metastasis of breast cancer by regulating the PI3K-SGK1-CTGF pathway through the NEDD4L-Smad2 axis (93). AG490, an inhibitor of the Janus tyrosine kinase 2 (JAK2), can promote human organic anion transporter-3 (hOAT3) ubiquitination and degradation by enhancing the binding of NEDD4L with hOAT3 and reducing NEDD4L phosphorylation (94). Wogonin, a natural flavonoid agent, was revealed to upregulate the expression of NEDD4L and suppress the PI3K/Akt pathway (95). Therefore, targeting NEDD4L with these compounds might be helpful for cancer therapy in the future.

\section{CONCLUSIONS}

In conclusion, NEDD4L suppresses the carcinogenesis by elevating the degradation of substrates of NEDD4L in most cancer types, while the role of NEDD4L in a few cancer types still remains controversial (Figure 2 and Table 1). Modulating upstream genes can affect the expression of NEDD4L and thus influence the progression of cancers (Figure 2 and Table 2). It is important to mention that NEDD4L has mutations, copy number variations $(\mathrm{CNV})$ gains, and $\mathrm{CNV}$ losses in a variety of human cancers (Figure 3). In some specific malignant tumors, 


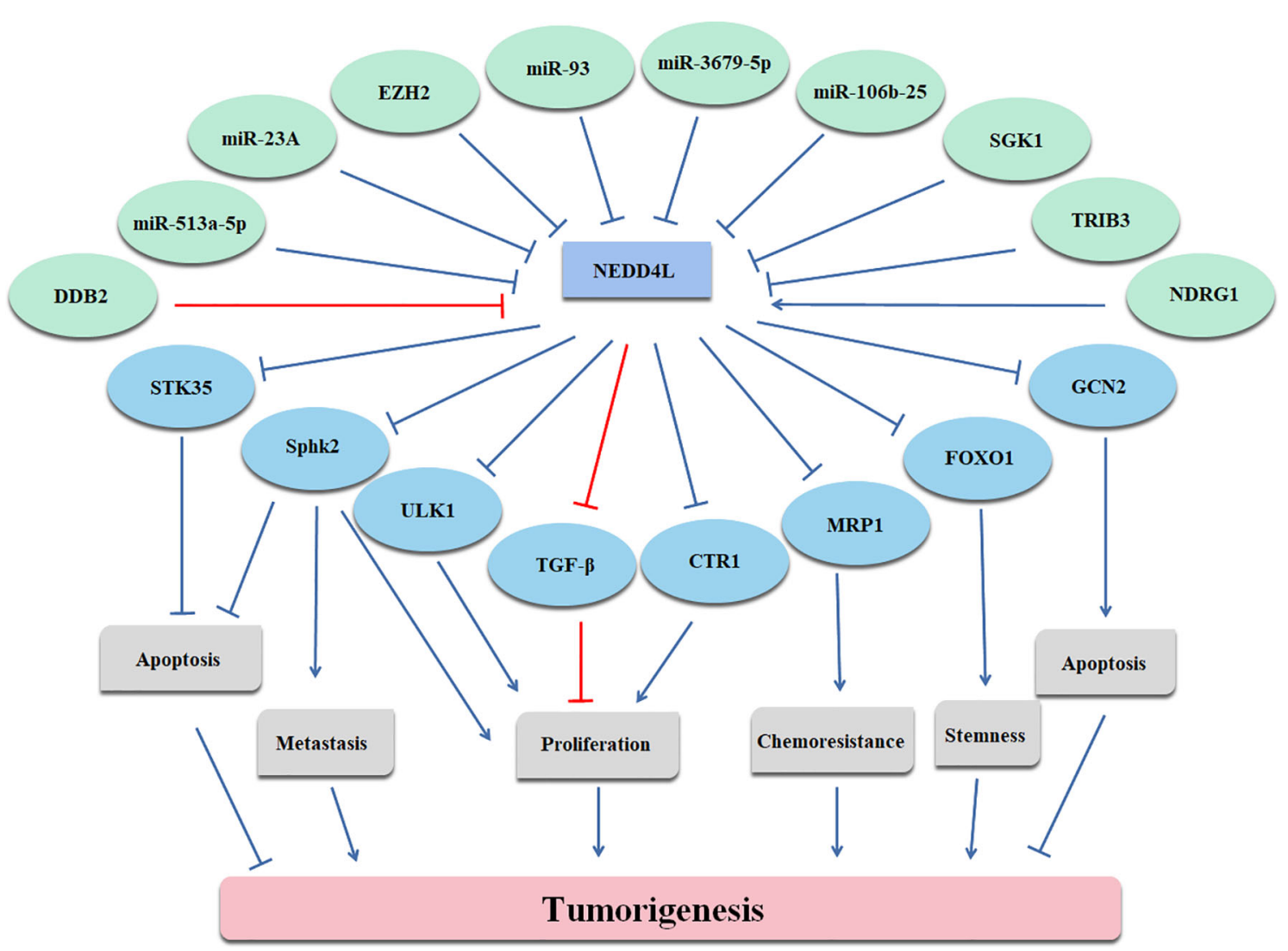

FIGURE 2 | Upstream and downstream genes of NEDD4L are illustrated.

TABLE 1 | NEDD4L gene modulates downstream genes and its impact on cancers.

\begin{tabular}{|c|c|c|c|}
\hline Cancer & Downstream gene & Function & Reference \\
\hline Colorectal cancer & STK35 & accelerates glycolysis, decreases apoptosis, attenuates chemosensitivity & $(42)$ \\
\hline Melanoma & STK35 & enhances $\mathrm{TH} 2$ differentiation & (84) \\
\hline \multirow[t]{2}{*}{ Pancreatic cancer } & LTF & binds to iron and transports iron & $(47)$ \\
\hline & ULK1 & involves in initiating cell autophagy & (30) \\
\hline \multirow[t]{2}{*}{ Lung cancer } & MRP1 & relates to multidrug resistance & (59) \\
\hline & GCN2 & increases the cell apoptosis & $(60)$ \\
\hline Breast cancer & $\mathrm{NOTCH} 1$ & takes part in $\mathrm{TIC}$ induction & (75) \\
\hline \multirow[t]{3}{*}{ Ovarian cancer } & TGF- $\beta$ & inhibits the cell proliferation & $(28)$ \\
\hline & FOXO1 & promotes breast cancer stemness & $(77)$ \\
\hline & CTR1 & is the predominant transporter of copper and elevates the development of cancer & (78) \\
\hline Glioma & SphK2 & increases the cell proliferation and EMT & (88) \\
\hline
\end{tabular}

TABLE 2 | Upstream gene modulates NEDD4L gene and its impact on cancers.

\begin{tabular}{lll}
\hline Cancer & Upstream gene & Function \\
\hline Pancreatic cancer & miR-23A & Reference \\
Nung cancer & EZH2 & $\begin{array}{l}\text { promotes proliferation, migration and invasiveness } \\
\text { suppresses iron-regulated metastasis } \\
\text { regulates cell cycle, apoptosis and metastasis } \\
\text { enhances TGF- } \beta \text {-induced EMT }\end{array}$ \\
miR-93 & stabilizes the c-Myc protein to induce chemoresistance \\
Ovarian cancer & miR-3679-5p & participates in gene transcription and cell cycle regulation \\
Breast cancer & mDB2 & takes part in TIC induction \\
Glioma & TRIB3 & possesses tumor initiation capacity
\end{tabular}



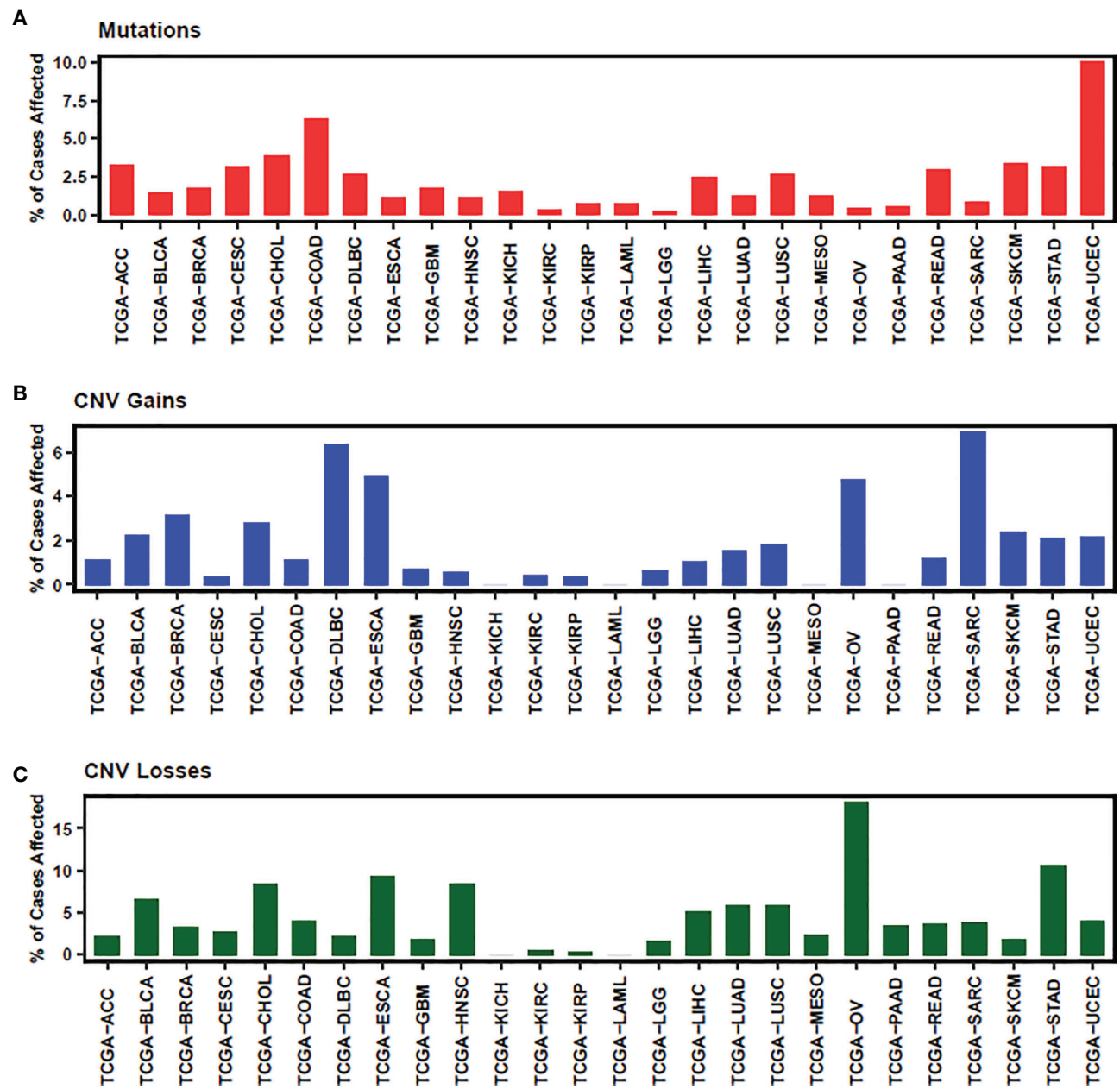

FIGURE 3 | The status of mutation (A), CNV gains (B) and CNV losses (C) of NEDD4L is illustrated. ACC, Adrenocortical carcinoma; BLCA, Bladder Urothelial Carcinoma; BRCA, Breast invasive carcinoma; CESC, Cervical squamous cell carcinoma and endocervical adenocarcinoma; CHOL, Cholangiocarcinoma; COAD, Colon adenocarcinoma; DLBC, Lymphoid Neoplasm Diffuse Large B-cell Lymphoma; ESCA, Esophageal carcinoma; GBM, Glioblastoma multiforme; HNSC, Head and Neck squamous cell carcinoma; KICH, Kidney Chromophobe; KIRC, Kidney renal clear cell carcinoma; KIRP, Kidney renal papillary cell carcinoma; LAML, Acute Myeloid Leukemia; LGG, Brain Lower Grade Glioma; LIHC, Liver hepatocellular carcinoma; LUAD, Lung adenocarcinoma; LUSC, Lung squamous cell carcinoma; MESO, Mesothelioma; OV, Ovarian serous cystadenocarcinoma; PAAD, Pancreatic adenocarcinoma; READ, Rectum adenocarcinoma; SARC, Sarcoma; SKCM, Skin Cutaneous Melanoma; STAD, Stomach adenocarcinoma; UCEC, Uterine Corpus Endometrial Carcinoma.

the design of the NEDD4L enhancer may contribute to the better treatment of cancer patients, but the targeted inhibition of NEDD4L probably controls the procedure of some other cancer types. Additionally, the concrete mechanisms have not been fully elucidated, although there are abundant studies proving that NEDD4L plays oncogenic or tumor-suppressive roles in cancers by modulating its substrates. More detailed mechanistic research should be carried out to fully understand the function and role of NEDD4L in tumorigenesis. It is also critical to design and develop NEDD4L enhancers and inhibitors for cancer treatment in patients with dysregulation of NEDD4L. We believe that the clinical application of NEDD4L enhancers or inhibitors in cancer therapy will be prospective in the near future.

\section{AUTHOR CONTRIBUTIONS}

SX and XZ wrote this manuscript. LX and HL searched the literature regarding NEDD4L and cancers. YS and SX prepared the figures and tables. Z-wW was critically involved in discussion. All authors contributed to the article and approved the submitted version. 


\section{REFERENCES}

1. Rieser E, Cordier SM, Walczak H. Linear Ubiquitination: A Newly Discovered Regulator of Cell Signalling. Trends Biochem Sci (2013) 38(2):94-102. doi: 10.1016/j.tibs.2012.11.007

2. Sun T, Liu Z, Yang Q. The Role of Ubiquitination and Deubiquitination in Cancer Metabolism. Mol Cancer (2020) 19(1):146. doi: 10.1186/s12943-020-01262-x

3. Hershko A, Ciechanover A. The Ubiquitin System. Annu Rev Biochem (1998) 67:425-79. doi: 10.1146/annurev.biochem.67.1.425

4. Mansour MA. Ubiquitination: Friend and Foe in Cancer. Int J Biochem Cell Biol (2018) 101:80-93. doi: 10.1016/j.biocel.2018.06.001

5. Weissman AM, Shabek N, Ciechanover A. The Predator Becomes the Prey: Regulating the Ubiquitin System by Ubiquitylation and Degradation. Nat Rev Mol Cell Biol (2011) 12(9):605-20. doi: 10.1038/nrm3173

6. Buetow L, Huang DT. Structural Insights Into the Catalysis and Regulation of E3 Ubiquitin Ligases. Nat Rev Mol Cell Biol (2016) 17(10):626-42. doi: 10.1038/nrm.2016.91

7. Qian H, Zhang Y, Wu B, Wu S, You S, Zhang N, et al. Structure and Function of HECT E3 Ubiquitin Ligases and Their Role in Oxidative Stress. J Transl Int Med (2020) 8(2):71-9. doi: 10.2478/jtim-2020-0012

8. Ciechanover A. The Ubiquitin-Proteasome Proteolytic Pathway. Cell (1994) 79(1):13-21. doi: 10.1016/0092-8674(94)90396-4

9. Wang ZW, Hu X, Ye M, Lin M, Chu M, Shen X. NEDD4 E3 Ligase: Functions and Mechanism in Human Cancer. Semin Cancer Biol (2020) 67(Pt 2):92101. doi: 10.1016/j.semcancer.2020.03.006

10. Ye X, Wang L, Shang B, Wang Z, Wei W. NEDD4: A Promising Target for Cancer Therapy. Curr Cancer Drug Targets (2014) 14(6):549-56. doi: 10.2174/ 1568009614666140725092430

11. Goel P, Manning JA, Kumar S. NEDD4-2 (NEDD4L): The Ubiquitin Ligase for Multiple Membrane Proteins. Gene (2015) 557(1):1-10. doi: 10.1016/ j.gene.2014.11.051

12. Ishigami T, Kino T, Minegishi S, Araki N, Umemura $M$, Ushio $H$, et al. Regulators of Epithelial Sodium Channels in Aldosterone-Sensitive Distal Nephrons (ASDN): Critical Roles of Nedd4L/Nedd4-2 and Salt-Sensitive Hypertension. Int J Mol Sci (2020) 21(11):3871. doi: 10.3390/ijms21113871

13. Zhao F, Gong X, Liu A, Lv X, Hu B, Zhang H. Downregulation of Nedd4L Predicts Poor Prognosis, Promotes Tumor Growth and Inhibits MAPK/ERK Signal Pathway in Hepatocellular Carcinoma. Biochem Biophys Res Commun (2018) 495(1):1136-43. doi: 10.1016/j.bbrc.2017.11.139

14. Takeuchi T, Adachi Y, Nagayama T, Furihata M. Nedd4L Modulates the Transcription of Metalloproteinase-1 and -13 Genes to Increase the Invasive Activity of Gallbladder Cancer. Int J Exp Pathol (2011) 92(2):79-86. doi: 10.1111/j.1365-2613.2010.00740.x

15. Yang B, Kumar S. Nedd4 and Nedd4-2: Closely Related Ubiquitin-Protein Ligases With Distinct Physiological Functions. Cell Death Differ (2010) 17 (1):68-77. doi: 10.1038/cdd.2009.84

16. Corbalan-Garcia S, Gomez-Fernandez JC. Signaling Through C2 Domains: More Than One Lipid Target. Biochim Biophys Acta (2014) 1838(6):1536-47. doi: 10.1016/j.bbamem.2014.01.008

17. Mari S, Ruetalo N, Maspero E, Stoffregen MC, Pasqualato S, Polo S, et al. Structural and Functional Framework for the Autoinhibition of Nedd4-Family Ubiquitin Ligases. Structure (2014) 22(11):1639-49. doi: 10.1016/j.str.2014.09.006

18. Todaro DR, Augustus-Wallace AC, Klein JM, Haas AL. The Mechanism of Neural Precursor Cell Expressed Developmentally Down-Regulated 4-2 (Nedd4-2)/NEDD4L-Catalyzed Polyubiquitin Chain Assembly. J Biol Chem (2017) 292(47):19521-36. doi: 10.1074/jbc.M117.817882

19. Zhu K, Shan Z, Chen X, Cai Y, Cui L, Yao W, et al. Allosteric Auto-Inhibition and Activation of the Nedd4 Family E3 Ligase Itch. EMBO Rep (2017) 18 (9):1618-30. doi: 10.15252/embr.201744454

20. Plant PJ, Yeger H, Staub O, Howard P, Rotin D. The C2 Domain of the Ubiquitin Protein Ligase Nedd4 Mediates Ca2+-Dependent Plasma Membrane Localization. J Biol Chem (1997) 272(51):32329-36. doi: 10.1074/jbc.272.51.32329

21. Ding Y, Zhang Y, Xu C, Tao QH, Chen YG. HECT Domain-Containing E3 Ubiquitin Ligase NEDD4L Negatively Regulates Wnt Signaling by Targeting Dishevelled for Proteasomal Degradation. J Biol Chem (2013) 288(12):828998. doi: 10.1074/jbc.M112.433185

22. Manning JA, Kumar S. Physiological Functions of Nedd4-2: Lessons From Knockout Mouse Models. Trends Biochem Sci (2018) 43(8):635-47. doi: 10.1016/j.tibs.2018.06.004
23. Kamynina E, Debonneville C, Bens M, Vandewalle A, Staub O. A Novel Mouse Nedd4 Protein Suppresses the Activity of the Epithelial Na+ Channel. FASEB J (2001) 15(1):204-14. doi: 10.1096/fj.00-0191com

24. Hughes JR, Parsons JL. The E3 Ubiquitin Ligase NEDD4L Targets OGG1 for Ubiquitylation and Modulates the Cellular DNA Damage Response. Front Cell Dev Biol (2020) 8:607060. doi: 10.3389/fcell.2020.607060

25. Gao P, Ma X, Yuan M, Yi Y, Liu G, Wen M, et al. E3 Ligase Nedd4l Promotes Antiviral Innate Immunity by Catalyzing K29-Linked Cysteine Ubiquitination of TRAF3. Nat Commun (2021) 12(1):1194. doi: 10.1038/s41467-021-21456-1

26. Kapoor S, Takeuchi T, Goto N, Kito Y, Furihata M. Role of Altered Expression of Nedd4L in the Pathogenesis of Systemic Malignancies. Int J Exp Pathol (2012) 93(6):463; author reply 463-4. doi: 10.1111/j.1365-2613.2012.00834.x

27. Persaud A, Alberts P, Amsen EM, Xiong X, Wasmuth J, Saadon Z, et al. Comparison of Substrate Specificity of the Ubiquitin Ligases Nedd4 and Nedd4-2 Using Proteome Arrays. Mol Syst Biol (2009) 5:333. doi: 10.1038/ msb. 2009.85

28. Zhao R, Cui T, Han C, Zhang X, He J, Srivastava AK, et al. DDB2 Modulates TGF-Beta Signal Transduction in Human Ovarian Cancer Cells by Downregulating NEDD4L. Nucleic Acids Res (2015) 43(16):7838-49. doi: $10.1093 /$ nar/gkv667

29. Nazio F, Cecconi F. Autophagy Up and Down by Outsmarting the Incredible ULK. Autophagy (2017) 13(5):967-8. doi: 10.1080/15548627.2017.1285473

30. Lee DE, Yoo JE, Kim J, Kim S, Kim S, Lee H, et al. NEDD4L Downregulates Autophagy and Cell Growth by Modulating ULK1 and a Glutamine Transporter. Cell Death Dis (2020) 11(1):38. doi: 10.1038/s41419-020-2242-5

31. Wang H, Sun RQ, Camera D, Zeng XY, Jo E, Chan SM, et al. Endoplasmic Reticulum Stress Up-Regulates Nedd4-2 to Induce Autophagy. FASEB $J$ (2016) 30(7):2549-56. doi: 10.1096/fj.201500119

32. Wang Z, Dang T, Liu T, Chen S, Li L, Huang S, et al. NEDD4L Protein Catalyzes Ubiquitination of PIK3CA Protein and Regulates PI3K-AKT Signaling. J Biol Chem (2016) 291(33):17467-77. doi: 10.1074/jbc.M116.726083

33. Gao S, Alarcon C, Sapkota G, Rahman S, Chen PY, Goerner N, et al. Ubiquitin Ligase Nedd4L Targets Activated Smad2/3 to Limit TGF-Beta Signaling. Mol Cell (2009) 36(3):457-68. doi: 10.1016/j.molcel.2009.09.043

34. Smyth EC, Nilsson M, Grabsch HI, van Grieken NC, Lordick F. Gastric Cancer. Lancet (2020) 396(10251):635-48. doi: 10.1016/S0140-6736(20) 31288-5

35. Gao C, Pang L, Ren C, Ma T. Decreased Expression of Nedd4L Correlates With Poor Prognosis in Gastric Cancer Patient. Med Oncol (2012) 29 (3):1733-8. doi: 10.1007/s12032-011-0061-3

36. Jiang $\mathrm{X}$, Zhang $\mathrm{S}$, Yin $\mathrm{Z}$, Sheng $\mathrm{Y}$, Yan $\mathrm{Q}$, Sun $\mathrm{R}$, et al. The Correlation Between NEDD4L and HIF-1alpha Levels as a Gastric Cancer Prognostic Marker. Int J Med Sci (2019) 16(11):1517-24. doi: 10.7150/ijms.34646

37. Sung H, Ferlay J, Siegel RL, Laversanne M, Soerjomataram I, Jemal A, et al. Global Cancer Statistics 2020: GLOBOCAN Estimates of Incidence and Mortality Worldwide for 36 Cancers in 185 Countries. CA Cancer J Clin (2021) 71(3):209-49. doi: 10.3322/caac.21660

38. Debebe A, Medina V, Chen CY, Mahajan IM, Jia C, Fu D, et al. Wnt/betaCatenin Activation and Macrophage Induction During Liver Cancer Development Following Steatosis. Oncogene (2017) 36(43):6020-9. doi: 10.1038/onc.2017.207

39. Lee HS, Park MH, Yang SJ, Park KC, Kim NS, Kim YS, et al. Novel Candidate Targets of Wnt/beta-Catenin Signaling in Hepatoma Cells. Life Sci (2007) 80 (7):690-8. doi: 10.1016/j.lfs.2006.10.024

40. Novellasdemunt L, Kucharska A, Jamieson C, Prange-Barczynska M, Baulies A, Antas P, et al. NEDD4 and NEDD4L Regulate Wnt Signalling and Intestinal Stem Cell Priming by Degrading LGR5 Receptor. EMBO J (2020) 39(3):e102771. doi: 10.15252/embj.2019102771

41. Tanksley JP, Chen X, Coffey RJ. NEDD4L is Downregulated in Colorectal Cancer and Inhibits Canonical WNT Signaling. PloS One (2013) 8(11):e81514. doi: 10.1371/journal.pone.0081514

42. Yang H, Zhu J, Wang G, Liu H, Zhou Y, Qian J. STK35 Is Ubiquitinated by NEDD4L and Promotes Glycolysis and Inhibits Apoptosis Through Regulating the AKT Signaling Pathway, Influencing Chemoresistance of Colorectal Cancer. Front Cell Dev Biol (2020) 8:582695. doi: 10.3389/ fcell. 2020.582695

43. Supiot S, Gouraud W, Campion L, Jezequel P, Buecher B, Charrier J, et al. Early Dynamic Transcriptomic Changes During Preoperative Radiotherapy in 
Patients With Rectal Cancer: A Feasibility Study. World J Gastroenterol (2013) 19(21):3249-54. doi: 10.3748/wjg.v19.i21.3249

44. Frampton AE, Castellano L, Colombo T, Giovannetti E, Krell J, Jacob J, et al. Integrated Molecular Analysis to Investigate the Role of microRNAs in Pancreatic Tumour Growth and Progression. Lancet (2015) 385(Suppl 1): S37. doi: 10.1016/S0140-6736(15)60352-X

45. Frampton AE, Castellano L, Colombo T, Giovannetti E, Krell J, Jacob J, et al. MicroRNAs Cooperatively Inhibit a Network of Tumor Suppressor Genes to Promote Pancreatic Tumor Growth and Progression. Gastroenterology (2014) 146(1):268-77 e18. doi: 10.1053/j.gastro.2013.10.010

46. Kovacevic Z, Chikhani S, Lui GY, Sivagurunathan S, Richardson DR. The Iron-Regulated Metastasis Suppressor NDRG1 Targets NEDD4L, PTEN, and SMAD4 and Inhibits the PI3K and Ras Signaling Pathways. Antioxid Redox Signal (2013) 18(8):874-87. doi: 10.1089/ars.2011.4273

47. Wang Y, Liu Y, Liu J, Kang R, Tang D. NEDD4L-Mediated LTF Protein Degradation Limits Ferroptosis. Biochem Biophys Res Commun (2020) 531 (4):581-7. doi: 10.1016/j.bbrc.2020.07.032

48. Sharma A, Sharma KL, Gupta A, Yadav A, Kumar A. Gallbladder Cancer Epidemiology, Pathogenesis and Molecular Genetics: Recent Update. World J Gastroenterol (2017) 23(22):3978-98. doi: 10.3748/wjg.v23.i22.3978

49. Bade BC, Dela Cruz CS. Lung Cancer 2020: Epidemiology, Etiology, and Prevention. Clin Chest Med (2020) 41(1):1-24. doi: 10.1016/j.ccm.2019.10.001

50. Wu J, Gao W, Tang Q, Yu Y, You W, Wu Z, et al. M2 Macrophage-Derived Exosomes Facilitate HCC Metastasis by Transferring alphaM Beta2 Integrin to Tumor Cells. Hepatology (2021) 73(4):1365-80. doi: 10.1002/hep.31432

51. Wang X, Duan J, Fu W, Yin Z, Sheng J, Lei Z, et al. Decreased Expression of NEDD4L Contributes to NSCLC Progression and Metastasis. Biochem Biophys Res Commun (2019) 513(2):398-404. doi: 10.1016/j.bbrc.2019.04.001

52. Sakashita $\mathrm{H}$, Inoue $\mathrm{H}$, Akamine $\mathrm{S}$, Ishida $\mathrm{T}$, Inase $\mathrm{N}$, Shirao $\mathrm{K}$, et al. Identification of the NEDD4L Gene as a Prognostic Marker by Integrated Microarray Analysis of Copy Number and Gene Expression Profiling in nonSmall Cell Lung Cancer. Ann Surg Oncol (2013) 20 Suppl 3:S590-8. doi: 10.1245/s10434-013-3059-6

53. Lu H, Li G, Zhou C, Jin W, Qian X, Wang Z, et al. Regulation and Role of Post-Translational Modifications of Enhancer of Zeste Homologue 2 in Cancer Development. Am J Cancer Res (2016) 6(12):2737-54.

54. Jiang $\mathrm{H}, \mathrm{Bu} \mathrm{Q}$, Zeng M, Xia D, Wu A. MicroRNA-93 Promotes Bladder Cancer Proliferation and Invasion by Targeting PEDF. Urol Oncol (2019) 37 (2):150-7. doi: 10.1016/j.urolonc.2018.08.001

55. Wang C, Tian S, Zhang D, Deng J, Cai H, Shi C, et al. Increased Expression of microRNA-93 Correlates With Progression and Prognosis of Prostate Cancer. Med (Baltimore) (2020) 99(22):e18432. doi: 10.1097/MD.0000000000018432

56. Li C, Lyu J, Meng QH. MiR-93 Promotes Tumorigenesis and Metastasis of Non-Small Cell Lung Cancer Cells by Activating the PI3K/Akt Pathway via Inhibition of LKB1/PTEN/CDKN1A. J Cancer (2017) 8(5):870-9. doi: $10.7150 /$ jca. 17958

57. Qu MH, Han C, Srivastava AK, Cui T, Zou N, Gao ZQ, et al. miR-93 Promotes TGF-Beta-Induced Epithelial-to-Mesenchymal Transition Through Downregulation of NEDD4L in Lung Cancer Cells. Tumour Biol (2016) 37 (4):5645-51. doi: 10.1007/s13277-015-4328-8

58. Wang H, Wang L, Pan H, Wang Y, Shi M, Yu H, et al. Exosomes Derived From Macrophages Enhance Aerobic Glycolysis and Chemoresistance in Lung Cancer by Stabilizing C-Myc via the Inhibition of NEDD4L. Front Cell Dev Biol (2020) 8:620603. doi: 10.3389/fcell.2020.620603

59. Lu KH, Broaddus RR. Endometrial Cancer. N Engl J Med (2020) 383 (21):2053-64. doi: 10.1056/NEJMra1514010

60. Wei C, Lin M, Jinjun B, Su F, Dan C, Yan C, et al. Involvement of General Control Nonderepressible Kinase 2 in Cancer Cell Apoptosis by Posttranslational Mechanisms. Mol Biol Cell (2015) 26(6):1044-57. doi: 10.1091/mbc.E14-10-1438

61. Yu G, Hsu WL, Coghill AE, Yu KJ, Wang CP, Lou PJ, et al. Whole-Exome Sequencing of Nasopharyngeal Carcinoma Families Reveals Novel Variants Potentially Involved in Nasopharyngeal Carcinoma. Sci Rep (2019) 9(1):9916. doi: 10.1038/s41598-019-46137-4

62. Lheureux S, Gourley C, Vergote I, Oza AM. Epithelial Ovarian Cancer. Lancet (2019) 393(10177):1240-53. doi: 10.1016/S0140-6736(18)32552-2

63. Yang Q, Zhao J, Cui M, Gi S, Wang W, Han X. Nedd4L Expression is Decreased in Ovarian Epithelial Cancer Tissues Compared to Ovarian non-
Cancer Tissue. J Obstet Gynaecol Res (2015) 41(12):1959-64. doi: 10.1111/ jog. 12808

64. Gilson P, Drouot G, Witz A, Merlin JL, Becuwe P, Harle A. Emerging Roles of DDB2 in Cancer. Int J Mol Sci (2019) 20(20):5168. doi: 10.3390/ijms20205168

65. Cui T, Srivastava AK, Han C, Wu D, Wani N, Liu L, et al. DDB2 Represses Ovarian Cancer Cell Dedifferentiation by Suppressing ALDH1A1. Cell Death Dis (2018) 9(5):561. doi: 10.1038/s41419-018-0585-y

66. Yilmaz E, Gul M, Melekoglu R, Inci Coskun E, Sahin N, Gul S, et al. Neural Precursor Cell-Expressed Developmentally Down-Regulated 4-Like: A New Biomarker in the Pathophysiology of Endometrial Cancer. J Int Med Res (2018) 46(9):3709-16. doi: 10.1177/0300060518777944

67. Hu XY, Xu YM, Fu Q, Yu JJ, Huang J. Nedd4L Expression is Downregulated in Prostate Cancer Compared to Benign Prostatic Hyperplasia. Eur J Surg Oncol (2009) 35(5):527-31. doi: 10.1016/j.ejso.2008.09.015

68. Qi H, Grenier J, Fournier A, Labrie C. Androgens Differentially Regulate the Expression of NEDD4L Transcripts in LNCaP Human Prostate Cancer Cells. Mol Cell Endocrinol (2003) 210(1-2):51-62. doi: 10.1016/j.mce.2003.08.009

69. Sherk AB, Frigo DE, Schnackenberg CG, Bray JD, Laping NJ, Trizna W, et al. Development of a Small-Molecule Serum- and Glucocorticoid-Regulated Kinase-1 Antagonist and its Evaluation as a Prostate Cancer Therapeutic. Cancer Res (2008) 68(18):7475-83. doi: 10.1158/0008-5472.CAN-08-1047

70. Wang Y, Qin T, Hu W, Chen B, Dai M, Xu G. Genome-Wide Methylation Patterns in Androgen-Independent Prostate Cancer Cells: A Comprehensive Analysis Combining MeDIP-Bisulfite, RNA, and microRNA Sequencing Data. Genes (Basel) (2018) 9(1):32. doi: 10.3390/genes9010032

71. Hellwinkel OJ, Asong LE, Rogmann JP, Sultmann H, Wagner C, Schlomm T, et al. Transcription Alterations of Members of the Ubiquitin-Proteasome Network in Prostate Carcinoma. Prostate Cancer Prostatic Dis (2011) 14 (1):38-45. doi: 10.1038/pcan.2010.48

72. Zhao H, Zhang J, Fu X, Mao D, Qi X, Liang S, et al. Integrated Bioinformatics Analysis of the NEDD4 Family Reveals a Prognostic Value of NEDD4L in Clear-Cell Renal Cell Cancer. PeerJ (2021) 9:e11880. doi: 10.7717/peerj.11880

73. Dong H, Zhu L, Sun J, Zhang Y, Cui Q, Wu L, et al. Pan-Cancer Analysis of NEDD4L and Its Tumor Suppressor Effects in Clear Cell Renal Cell Carcinoma. J Cancer (2021) 12(20):6242-53. doi: 10.7150/jca.58004

74. Iacoviello L, Bonaccio M, de Gaetano G, Donati MB. Epidemiology of Breast Cancer, a Paradigm of the "Common Soil" Hypothesis. Semin Cancer Biol (2021) 72:4-10. doi: 10.1016/j.semcancer.2020.02.010

75. Guarnieri AL, Towers CG, Drasin DJ, Oliphant MUJ, Andrysik Z, Hotz TJ, et al. The miR-106b-25 Cluster Mediates Breast Tumor Initiation Through Activation of NOTCH1 via Direct Repression of NEDD4L. Oncogene (2018) 37(28):3879-93. doi: 10.1038/s41388-018-0239-7

76. Pires BR, DEA IS, Souza LD, Rodrigues JA, Mencalha AL. Targeting Cellular Signaling Pathways in Breast Cancer Stem Cells and its Implication for Cancer Treatment. Anticancer Res (2016) 36(11):5681-91. doi: 10.21873/ anticanres. 11151

77. Yu JM, Sun W, Wang ZH, Liang X, Hua F, Li K, et al. TRIB3 Supports Breast Cancer Stemness by Suppressing FOXO1 Degradation and Enhancing SOX2 Transcription. Nat Commun (2019) 10(1):5720. doi: 10.1038/s41467-01913700-6

78. Guo J, Cheng J, Zheng N, Zhang X, Dai X, Zhang L, et al. Copper Promotes Tumorigenesis by Activating the PDK1-AKT Oncogenic Pathway in a Copper Transporter 1 Dependent Manner. Adv Sci (Weinh) (2021) 18:e2004303. doi: $10.1002 /$ advs. 202004303

79. Sandsveden M, Nilsson E, Borgquist S, Rosendahl AH, Manjer J. Prediagnostic Serum Selenium Levels in Relation to Breast Cancer Survival and Tumor Characteristics. Int J Cancer (2020) 147(9):2424-36. doi: 10.1002/ijc.33031

80. Liang ZZ, Zhu RM, Li YL, Jiang HM, Li RB, Wang Q, et al. Differential Epigenetic Profiles Induced by Sodium Selenite in Breast Cancer Cells. J Trace Elem Med Biol (2021) 64:126677. doi: 10.1016/j.jtemb.2020.126677

81. Hasna J, Hague F, Rodat-Despoix L, Geerts D, Leroy C, Tulasne D, et al. Orai3 Calcium Channel and Resistance to Chemotherapy in Breast Cancer Cells: The P53 Connection. Cell Death Differ (2018) 25(4):693-707. doi: 10.1038/ s41418-017-0007-1

82. Mathieu NA, Levin RH, Spratt DE. Exploring the Roles of HERC2 and the NEDD4L HECT E3 Ubiquitin Ligase Subfamily in P53 Signaling and the DNA Damage Response. Front Oncol (2021) 11:659049. doi: 10.3389/ fonc.2021.659049 
83. Cui J, Shu C, Xu J, Chen D, Li J, Ding K, et al. JP1 Suppresses Proliferation and Metastasis of Melanoma Through MEK1/2 Mediated NEDD4L-SP1-Integrin Alphavbeta3 Signaling. Theranostics (2020) 10(18):8036-50. doi: 10.7150/ thno. 45843

84. Heikamp EB, Patel CH, Collins S, Waickman A, Oh MH, Sun IH, et al. The AGC Kinase SGK1 Regulates TH1 and TH2 Differentiation Downstream of the Mtorc2 Complex. Nat Immunol (2014) 15(5):457-64. doi: 10.1038/ni.2867

85. Kito Y, Bai J, Goto N, Okubo H, Adachi Y, Nagayama T, et al. Pathobiological Properties of the Ubiquitin Ligase Nedd4L in Melanoma. Int J Exp Pathol (2014) 95(1):24-8. doi: 10.1111/iep.12051

86. He S, Deng J, Li G, Wang B, Cao Y, Tu Y. Down-Regulation of Nedd4L is Associated With the Aggressive Progression and Worse Prognosis of Malignant Glioma. Jpn J Clin Oncol (2012) 42(3):196-201. doi: 10.1093/jjco/ hyr195

87. Chen KC, Chen PH, Ho KH, Shih CM, Chou CM, Cheng CH, et al. IGF-1Enhanced miR-513a-5p Signaling Desensitizes Glioma Cells to Temozolomide by Targeting the NEDD4L-Inhibited Wnt/beta-Catenin Pathway. PloS One (2019) 14(12):e0225913. doi: 10.1371/journal.pone.0225913

88. Wang H, Zhao B, Bian E, Zong G, He J, Wang Y, et al. Ubiquitination Destabilizes Protein Sphingosine Kinase 2 to Regulate Glioma Malignancy. Front Cell Neurosci (2021) 15:660354. doi: 10.3389/fncel.2021.660354

89. Booken N, Gratchev A, Utikal J, Weiss C, Yu X, Qadoumi M, et al. Sezary Syndrome is a Unique Cutaneous T-Cell Lymphoma as Identified by an Expanded Gene Signature Including Diagnostic Marker Molecules CDO1 and DNM3. Leukemia (2008) 22(2):393-9. doi: 10.1038/sj.leu.2405044

90. Qu Q, Li Y, Fang X, Zhang L, Xue C, Ge X, et al. Differentially Expressed tRFs in CD5 Positive Relapsed \& Refractory Diffuse Large B Cell Lymphoma and the Bioinformatic Analysis for Their Potential Clinical Use. Biol Direct (2019) 14(1):23. doi: 10.1186/s13062-019-0255-8

91. Zhang X, He Y, Gu H, Liu Z, Li B, Yang Y, et al. Construction of a NineMicroRNA-Based Signature to Predict the Overall Survival of Esophageal Cancer Patients. Front Genet (2021) 12:670405. doi: 10.3389/fgene.2021. 670405
92. Zhang W, Zhang R, Zeng Y, Li Y, Chen Y, Zhou J, et al. ALCAP2 Inhibits Lung Adenocarcinoma Cell Proliferation, Migration and Invasion via the Ubiquitination of Beta-Catenin by Upregulating the E3 Ligase NEDD4L. Cell Death Dis (2021) 12(8):755. doi: 10.1038/s41419-021-04043-6

93. Zhang Y, Shi G, Zhang H, Xiong Q, Cheng F, Wang H, et al. Dexamethasone Enhances the Lung Metastasis of Breast Cancer via a PI3K-SGK1-CTGF Pathway. Oncogene (2021) 40(35):5367-78. doi: 10.1038/s41388-021-01944-w

94. Zhang J, Liu C, You G. AG490, a JAK2-Specific Inhibitor, Downregulates the Expression and Activity of Organic Anion Transporter-3. J Pharmacol Sci (2018) 136(3):142-8. doi: 10.1016/j.jphs.2018.01.006

95. Qian W, Yu D, Zhang J, Hu Q, Tang C, Liu P, et al. Wogonin Attenuates Isoprenaline-Induced Myocardial Hypertrophy in Mice by Suppressing the PI3K/ Akt Pathway. Front Pharmacol (2018) 9:896. doi: 10.3389/fphar.2018.00896

96. Wang W, Ning JZ, Tang ZG, He Y, Yao LC, Ye L, et al. MicroRNA-23a Acts as an Oncogene in Pancreatic Carcinoma by Targeting TFPI-2. Exp Ther Med (2020) 20(5):53. doi: 10.3892/etm.2020.9181

Conflict of Interest: The authors declare that the research was conducted in the absence of any commercial or financial relationships that could be construed as a potential conflict of interest.

Publisher's Note: All claims expressed in this article are solely those of the authors and do not necessarily represent those of their affiliated organizations, or those of the publisher, the editors and the reviewers. Any product that may be evaluated in this article, or claim that may be made by its manufacturer, is not guaranteed or endorsed by the publisher.

Copyright (c) 2021 Xie, Xia, Song, Liu, Wang and Zhu. This is an open-access article distributed under the terms of the Creative Commons Attribution License (CC BY). The use, distribution or reproduction in other forums is permitted, provided the original author(s) and the copyright owner(s) are credited and that the original publication in this journal is cited, in accordance with accepted academic practice. No use, distribution or reproduction is permitted which does not comply with these terms. 